KYUNGPOOK Math. J. 53(2013), 207-218

http://dx.doi.org/10.5666/KMJ.2013.53.2.207

\title{
Integrability of Distributions in GCR-lightlike Submanifolds of Indefinite Sasakian Manifolds
}

VARUN JAIN

Department of Mathematics, Multani Mal Modi College, Patiala 147001, India

e-mail : varun82jain@gmail.com

RAKESH KUMAR*

University College of Engineering, Punjabi University, Patiala 147002, India

e-mail : dr_rk37c@yahoo.co.in

RAKESh Kumar NAGAich

Department of Mathematics, Punjabi University, Patiala 147002, India

e-mail : nagaichrakesh@yahoo.com

ABSTRACT. In this paper, we study $G C R$-lightlike submanifolds of indefinite Sasakian manifold. We give some necessary and sufficient conditions on integrability of various distributions of $G C R$-lightlike submanifold of an indefinite Sasakian manifold. We also find the conditions for each leaf of holomorphic distribution and radical distribution is totally geodesic.

\section{Introduction}

The geometry of $C R$-submanifolds of Kaehler manifolds were introduced by Bejancu [2] in 1978 and further developed by Bejancu [3, 4], Chen [5, 6], Duggal $[8,9]$, Yano and Kon $[16,18]$ and others. Contact $C R$-submanifolds of Sasakian manifolds with definite metric were introduced by Yano and Kon [17] in 1982. For indefinite metric, Duggal and Sahin [11] introduced the notion of contact $C R$ lightlike submanifolds of indefinite Sasakian manifolds. In continuation, recently Duggal and Sahin [12] introduced a new class of submanifolds namely generalized Cauchy-Riemannian $(G C R)$-lightlike submanifolds of indefinite Sasakian manifolds, which is an umbrella of invariant, screen real, contact $C R$-lightlike submanifolds. Since significant applications of contact geometry (Arnol'd [1], Nazaikinskii et. al. [14], Maclane [15]) with definite and indefinite metric and limited information available on its $G C R$-lightlike case motivated us to extend the work of them. After the

* Corresponding Author.

Received February 24, 2011; revised November 30, 2011; accepted September 7, 2012.

2010 Mathematics Subject Classification: 53C15, 53C40, 53C50.

Key words and phrases: Lightlike submanifold, indefinite Sasakian manifolds, $G C R$ lightlike submanifold. 
brief information of $G C R$-lightlike submanifolds of indefinite Sasakian manifolds, we study the integrability of various distributions.

\section{Lightlike Submanifolds}

We recall notations and fundamental equations for lightlike submanifolds, which are due to the book [10] by Duggal and Bejancu. A submanifold $\left(M^{m}, g\right)$ immersed in a semi-Riemannian manifold $\left(\bar{M}^{m+n}, \bar{g}\right)$ is called a $r$-lightlike submanifold if the metric $g$ induced from $\bar{g}$ is degenerate and the radical distribution $\operatorname{Rad}(T M)$ is of rank $r$, where $1 \leq r \leq m$. Let $S(T M)$ be a screen distribution which is a semi-Riemannian complementary distribution of $\operatorname{Rad}(T M)$ in $T M$, that is, $T M=\operatorname{Rad}(T M) \perp S(T M)$. Consider a screen transversal vector bundle $S\left(T M^{\perp}\right)$, which is a semi-Riemannian complementary vector bundle of $\operatorname{Rad}(T M)$ in $T M^{\perp}$. Since, for any local basis $\left\{\xi_{i}\right\}$ of $\operatorname{Rad}(T M)$, there exists a local null frame $\left\{N_{i}\right\}$ of sections with values in the orthogonal complement of $S\left(T M^{\perp}\right)$ in $(S(T M))^{\perp}$ such that $\bar{g}\left(\xi_{i}, N_{j}\right)=$ ?delta $a_{i j}$, it follows that there exists a lightlike transversal vector bundle $\operatorname{lt}(T M)$ locally spanned by $\left\{N_{i}\right\}$. Let $\operatorname{tr}(T M)$ be complementary (but not orthogonal) vector bundle to $T M$ in $T \bar{M}$. Then,

$$
\left.T \bar{M}\right|_{M}=T M \oplus \operatorname{tr}(T M)=(\operatorname{Rad} T M \oplus \operatorname{lt}(T M)) \perp S(T M) \perp S\left(T M^{\perp}\right) .
$$

Let $\bar{\nabla}$ be the Levi-Civita connection on $\bar{M}$. Then according to the decomposition (2.1), the Gauss and Weingarten formulas are given by

$$
\begin{gathered}
\bar{\nabla}_{X} Y=\nabla_{X} Y+h(X, Y), \quad \forall \quad X, Y \in \Gamma(T M), \\
\bar{\nabla}_{X} U=-A_{U} X+\nabla_{X}^{\perp} U, \quad \forall \quad X \in \Gamma(T M), \quad U \in \Gamma(\operatorname{tr}(T M)),
\end{gathered}
$$

where $\left\{\nabla_{X} Y, A_{U} X\right\}$ and $\left\{h(X, Y), \nabla_{X}^{\perp} U\right\}$ belong to $\Gamma(T M)$ and $\Gamma(\operatorname{tr}(T M))$, respectively. Here $\nabla$ is a torsion-free linear connection on $M, h$ is a symmetric bilinear form on $\Gamma(T M)$ which is called a second fundamental form, $A_{U}$ is linear a operator on $M$ and known as a shape operator. Considering the projection morphisms $L$ and $S$ of $\operatorname{tr}(T M)$ on $\operatorname{ltr}(T M)$ and $S\left(T M^{\perp}\right)$, respectively, then (2.2) and (2.3) give

$$
\begin{aligned}
& \bar{\nabla}_{X} Y=\nabla_{X} Y+h^{l}(X, Y)+h^{s}(X, Y), \\
& \bar{\nabla}_{X} N=-A_{N} X+\nabla_{X}^{l} N+D^{s}(X, N), \\
& \bar{\nabla}_{X} W=-A_{W} X+\nabla_{X}^{s} W+D^{l}(X, W),
\end{aligned}
$$

where $X \in \Gamma(T M), \quad N \in \Gamma(\operatorname{ltr}(T M))$ and $W \in \Gamma\left(S\left(T M^{\perp}\right)\right)$. By using (2.4)-(2.5), we obtain

$$
\bar{g}\left(h^{s}(X, Y), W\right)+\bar{g}\left(Y, D^{l}(X, W)\right)=g\left(A_{W} X, Y\right)
$$




$$
\bar{g}\left(h^{l}(X, Y), \xi\right)+\bar{g}\left(Y, h^{l}(X, \xi)\right)+g\left(Y, \nabla_{X} \xi\right)=0,
$$

for any $\xi \in \Gamma(\operatorname{Rad} T M)$ and $W \in \Gamma\left(S\left(T M^{\perp}\right)\right)$.

Let $P$ be the projection morphism of $T M$ on $S(T M)$. Then we can induce some new geometric objects on the screen distribution $S(T M)$ on $M$ as

$$
\begin{gathered}
\nabla_{X} P Y=\nabla_{X}^{*} P Y+h^{*}(X, Y), \\
\nabla_{X} \xi=-A_{\xi}^{*} X+\nabla_{X}^{* t} \xi
\end{gathered}
$$

for any $X, Y \in \Gamma(T M)$ and $\xi \in \Gamma(\operatorname{RadTM})$, where $\left\{\nabla_{X}^{*} P Y, A_{\xi}^{*} X\right\}$ and $\left\{h^{*}(X, Y), \nabla_{X}^{* t} \xi\right\}$ belong to $\Gamma(S(T M))$ and $\Gamma(\operatorname{Rad} T M)$, respectively.

Next, we recall some basic definitions and results of indefinite Sasakian manifolds from [13]. An odd dimensional semi-Riemannian manifold $(\bar{M}, \bar{g})$ is called an $\epsilon$-contact metric manifold, if there is a $(1,1)$ tensor field $\phi$, a vector field $V$ and a 1-form $\eta$ such that

$$
\begin{gathered}
\bar{g}(\phi X, \phi Y)=\bar{g}(X, Y)-\epsilon \eta(X) \eta(Y), \quad \bar{g}(V, V)=\epsilon, \\
\phi^{2}(X)=-X+\eta(X) V, \quad \bar{g}(X, V)=\epsilon \eta(X), \\
d \eta(X, Y)=\bar{g}(X, \phi Y), \forall X, Y \in \Gamma(T M), \text { where } \quad \epsilon= \pm 1 .
\end{gathered}
$$

It follows that

$$
\phi V=0, \quad \eta \circ \phi=0, \quad \eta(V)=1 .
$$

Then $(\phi, V, \eta, \bar{g})$ is called an $\epsilon$-contact metric structure of $\bar{M}$. We say that $\bar{M}$ has a normal contact structure if $N_{\phi}+d \eta \otimes V=0$, where $N_{\phi}$ is Nijenhuis tensor field of $\phi$. A normal $\epsilon$-contact metric manifold is called an $\epsilon$-Sasakian manifold. For which we have

$$
\bar{\nabla}_{X} V=\phi X, \quad\left(\bar{\nabla}_{X} \phi\right) Y=-\bar{g}(X, Y) V+\epsilon \eta(Y) X
$$

\section{Generalized Cauchy-Riemann $(G C R)$-lightlike submanifolds}

Calin[7] proved that if the characteristic vector field $V$ is tangent to $(M, g, S(T M))$ then it belongs to $S(T M)$. We assume that the characteristic vector $V$ is tangent to $M$ throughout this paper.

Definition 2.2. ([12]). Let $(M, g, S(T M))$ be a real lightlike submanifold of an indefinite Sasakian manifold $(\bar{M}, \bar{g})$ then $M$ is called a generalized Cauchy-Riemann $(G C R)$-lightlike submanifold if the following conditions are satisfied 
(A) There exist two subbundles $D_{1}$ and $D_{2}$ of $\operatorname{Rad}(T M)$ such that

$$
\operatorname{Rad}(T M)=D_{1} \oplus D_{2}, \quad \phi\left(D_{1}\right)=D_{1}, \quad \phi\left(D_{2}\right) \subset S(T M) .
$$

(B) There exist two subbundles $D_{0}$ and $\bar{D}$ of $S(T M)$ such that

$$
S(T M)=\left\{\phi D_{2} \oplus \bar{D}\right\} \perp D_{0} \perp V, \quad \phi(\bar{D})=L \perp S .
$$

where $D_{0}$ is an invariant non degenerate distribution on $M,\{V\}$ is one dimensional distribution spanned by $V$ and $L, S$ are vector subbundles of $\operatorname{ltr}(T M)$ and $S(T M)^{\perp}$, respectively.

Then tangent bundle $T M$ of $M$ is decomposed as

$$
T M=\{D \oplus \bar{D} \oplus\{V\}\}, \quad D=\operatorname{Rad}(T M) \oplus D_{0} \oplus \phi\left(D_{2}\right) .
$$

An $r$-lightlike submanifold $M$ is called a proper $G C R$-lightlike submanifold if $D_{1} \neq$ $0, D_{2} \neq 0, D_{0} \neq 0$ and $S \neq 0$. Let $Q, P_{1}, P_{2}$ be the projection morphism on $D$, $\phi S \subset \bar{D}, \phi L \subset \bar{D}$ respectively, therefore

$$
X=Q X+V+P_{1} X+P_{2} X,
$$

for $X \in \Gamma(T M)$. Applying $\phi$ to (3.1), we obtain

$$
\phi X=f X+\omega P_{1} X+\omega P_{2} X,
$$

where $f X \in \Gamma(D), \omega P_{1} X \in \Gamma(S)$ and $\omega P_{2} X \in \Gamma(L)$ or we can write (3.2) as

$$
\phi X=f X+\omega X,
$$

where $f X$ and $\omega X$ are the tangential and transversal components of $\phi X$, respectively.

Similarly

$$
\phi U=B U+C U, \quad U \in \Gamma(\operatorname{tr}(T M)),
$$

where $B U$ and $C U$ are the sections of $T M$ and $\operatorname{tr}(T M)$, respectively. Differentiating (3.2) and using (2.5)-(2.7), (2.8) and (3.3), we have

$$
\begin{aligned}
& D^{l}\left(X, \omega P_{1} Y\right)=-\nabla_{X}^{l} \omega P_{2} Y+\omega P_{2} \nabla_{X} Y-h^{l}(X, f Y)+C h^{l}(X, Y) . \\
& D^{s}\left(X, \omega P_{2} Y\right)=-\nabla_{X}^{s} \omega P_{1} Y+\omega P_{1} \nabla_{X} Y-h^{s}(X, f Y)+C h^{s}(X, Y),
\end{aligned}
$$

for all $X, Y \in \Gamma(T M)$.

\section{Integrability of Distributions}

Let $\bar{M}$ be a real $m+n$-dimensional smooth manifold then a distribution of 
rank $t$ on $\bar{M}$ is a mapping $D$ defined on $\bar{M}$, which assign to each point $x$ of $\bar{M}$ a $t$-dimensional linear subspace $D_{x}$ of $T_{x}(M)$. A vector field $X$ on $\bar{M}$ is said to belong to $D$ if $X(x) \in D_{x}$ for each $x$ of $\bar{M}$. The distribution $D$ is said to be involutive if $[X, Y] \in \Gamma(D)$, for any $X, Y \in \Gamma(D)$. Then from page no. 34 of [10], a distribution $D$ is integrable if and only if it is involutive.

Theorem 4.1. Let $M$ be a proper GCR-lightlike submanifold of an indefinite Sasakian manifold $\bar{M}$. Then $\bar{D}$ is integrable if and only if $\nabla_{X} \phi Y=\nabla_{Y} \phi X$ for any $X, Y \in \Gamma(\bar{D})$.

Proof. For any $X, Y \in \Gamma(\bar{D})$ we have

$$
h(X, \phi Y)=\bar{\nabla}_{X} \phi Y-\nabla_{X} \phi Y .
$$

Replacing $X$ by $Y$ and subtracting the resulting equation from the above equation, we get

$$
\begin{aligned}
h(X, \phi Y)-h(Y, \phi X) & =\bar{\nabla}_{X} \phi Y-\bar{\nabla}_{Y} \phi X-\nabla_{X} \phi Y+\nabla_{Y} \phi X \\
& =\phi[X, Y]-\nabla_{X} \phi Y+\nabla_{Y} \phi X \\
& =f[X, Y]+\omega[X, Y]-\nabla_{X} \phi Y+\nabla_{Y} \phi X .
\end{aligned}
$$

Taking tangential parts of this equation, we have

$$
f[X, Y]=\nabla_{X} \phi Y-\nabla_{Y} \phi X .
$$

Hence from (4.1), the result follows.

Theorem 4.2. Let proper $M$ be a GCR-lightlike submanifold of an indefinite Sasakian manifold $\bar{M}$. Then $D_{0}$ is integrable if and only if

(i) $\bar{g}\left(h^{*}(X, Y), N\right)=\bar{g}\left(h^{*}(Y, X), N\right)$

(ii) $g\left(\nabla_{X}^{*} Y, V\right)=g\left(\nabla_{Y}^{*} X, V\right)$

(iii) $\bar{g}\left(h^{*}(X, \phi Y), N_{1}\right)=\bar{g}\left(h^{*}(Y, \phi X), N_{1}\right)$

(iv) $h^{s}(X, \phi Y)=h^{s}(Y, \phi X)$

(v) $g\left(\nabla_{X}^{*} Y, \phi \xi\right)=g\left(\nabla_{Y}^{*} X, \phi \xi\right)$,

for any $X, Y \in \Gamma\left(D_{0}\right), N \in \Gamma(\operatorname{ltr}(T M)), N_{1} \in \Gamma(L), W \in \Gamma(S)$, and $\xi \in \Gamma\left(D_{2}\right)$.

Proof. Using the definition of $G C R$-lightlike submanifold $D_{0}$ is integrable if and only if

$\bar{g}([X, Y], \phi W)=\bar{g}([X, Y], \phi \xi)=\bar{g}([X, Y], V)=\bar{g}\left([X, Y], \phi N_{1}\right)=\bar{g}([X, Y], N)=0$,

for any $X, Y \in \Gamma\left(D_{0}\right), N \in \Gamma(l \operatorname{tr}(T M)), N_{1} \in \Gamma(L), W \in \Gamma(S)$, and $\xi \in \Gamma\left(D_{2}\right)$.

Using (2.9) we have

$$
\bar{g}([X, Y], N)=\bar{g}\left(h^{*}(X, Y), N\right)-\bar{g}\left(h^{*}(Y, X), N\right),
$$


and

$$
\bar{g}([X, Y], V)=g\left(\nabla_{X}^{*} Y, V\right)-g\left(\nabla_{Y}^{*} X, V\right)
$$

On the other hand using (2.4) and (2.14) we get

$$
\begin{aligned}
\bar{g}\left([X, Y], \phi N_{1}\right) & =\bar{g}\left(\bar{\nabla}_{X} Y, \phi N_{1}\right)-\bar{g}\left(\bar{\nabla}_{Y} X, \phi N_{1}\right) \\
& =-\bar{g}\left(\nabla_{X} \phi Y, N_{1}\right)+\bar{g}\left(\nabla_{Y} \phi X, N_{1}\right) \\
& =-\bar{g}\left(h^{*}(X, \phi Y), N_{1}\right)+\bar{g}\left(h^{*}(Y, \phi X), N_{1}\right) .
\end{aligned}
$$

From (2.4) and (2.14) and using the indefinite Sasakian character of $\bar{M}$, we derive

$$
\begin{aligned}
\bar{g}([X, Y], \phi W) & =\bar{g}\left(\bar{\nabla}_{X} Y, \phi W\right)-\bar{g}\left(\bar{\nabla}_{Y} X, \phi W\right) \\
& =-\bar{g}\left(\bar{\nabla}_{X} \phi Y, W\right)+\bar{g}\left(\bar{\nabla}_{Y} \phi X, W\right) \\
& =-\bar{g}\left(h^{s}(X, \phi Y), W\right)+\bar{g}\left(h^{s}(Y, \phi X), W\right) .
\end{aligned}
$$

Finally from equation (2.4) and (2.9) we obtain

$$
\begin{aligned}
\bar{g}([X, Y], \phi \xi) & =g\left(\nabla_{X} Y, \phi \xi\right)-g\left(\nabla_{Y} X, \phi \xi\right) \\
& =g\left(\nabla_{X}^{*} Y, \phi \xi\right)-g\left(\nabla_{Y}^{*} X, \phi \xi\right) .
\end{aligned}
$$

Thus from (4.2)-(4.6) the proof is complete.

Theorem 4.3. Let $M$ be a proper GCR-lightlike submanifold of an indefinite Sasakian manifold $\bar{M}$. Then RadTM is integrable if and only if

(i) $g\left(A_{\xi^{\prime \prime}}^{*} \xi^{\prime}, V\right)=g\left(A_{\xi^{\prime}}^{*} \xi^{\prime \prime}, V\right)$

(ii) $\bar{g}\left(h^{l}\left(\xi^{\prime}, Z\right), \xi^{\prime \prime}\right)=\bar{g}\left(h^{l}\left(\xi^{\prime \prime}, Z\right), \xi^{\prime}\right)$

(iii) $\bar{g}\left(h^{l}\left(\xi^{\prime}, \phi \xi\right), \xi^{\prime \prime}\right)=\bar{g}\left(h^{l}\left(\xi^{\prime \prime}, \phi \xi\right), \xi^{\prime}\right)$

(iv) $h^{s}\left(\xi^{\prime}, \phi \xi^{\prime \prime}\right)=h^{s}\left(\xi^{\prime \prime}, \phi \xi^{\prime}\right)$

(v) $g\left(h^{*}\left(\xi^{\prime}, \phi \xi^{\prime \prime}\right), N_{1}\right)=g\left(h^{*}\left(\xi^{\prime \prime}, \phi \xi^{\prime}\right), N_{1}\right)$.

for any $\xi \in \Gamma\left(D_{2}\right), \xi^{\prime}, \xi^{\prime \prime} \in \Gamma(\operatorname{Rad}(T M)), N_{1} \in \Gamma(L), W \in \Gamma(S), Z \in \Gamma\left(D_{0}\right)$.

Proof. Using the definition of $G C R$-lightlike submanifold of an indefinite Sasakian manifold, $\operatorname{Rad}(T M)$ is integrable if and only if

$\bar{g}\left(\left[\xi^{\prime}, \xi^{\prime \prime}\right], V\right)=\bar{g}\left(\left[\xi^{\prime}, \xi^{\prime \prime}\right], Z\right)=\bar{g}\left(\left[\xi^{\prime}, \xi^{\prime \prime}\right], \phi \xi\right)=\bar{g}\left(\left[\xi^{\prime}, \xi^{\prime \prime}\right], \phi W\right)=\bar{g}\left(\left[\xi^{\prime}, \xi^{\prime \prime}\right], \phi N_{1}\right)=0$, for any $\xi \in \Gamma\left(D_{2}\right), \xi^{\prime}, \xi^{\prime \prime} \in \Gamma(\operatorname{Rad}(T M)), N_{1} \in \Gamma(L), W \in \Gamma(S)$, and $Z \in \Gamma\left(D_{0}\right)$. Using (2.10), we obtain

$$
\bar{g}\left(\left[\xi^{\prime}, \xi^{\prime \prime}\right], V\right)=-g\left(A_{\xi^{\prime \prime}}^{*} \xi^{\prime}, V\right)+g\left(A_{\xi^{\prime}}^{*} \xi^{\prime \prime}, V\right),
$$


and

$$
\begin{aligned}
\bar{g}\left(\left[\xi^{\prime}, \xi^{\prime \prime}\right], Z\right) & =-g\left(A_{\xi^{\prime \prime}}^{*} \xi^{\prime}, Z\right)+g\left(A_{\xi^{\prime}}^{*} \xi^{\prime \prime}, Z\right) \\
& =-\bar{g}\left(h^{l}\left(\xi^{\prime}, Z\right), \xi^{\prime \prime}\right)+\bar{g}\left(h^{l}\left(\xi^{\prime \prime}, Z\right), \xi^{\prime}\right) .
\end{aligned}
$$

Using (2.4) we get

$$
\begin{aligned}
\bar{g}\left(\left[\xi^{\prime}, \xi^{\prime \prime}\right], \phi \xi\right) & =\bar{g}\left(\bar{\nabla}_{\xi^{\prime}} \xi^{\prime \prime}, \phi \xi\right)-\bar{g}\left(\bar{\nabla}_{\xi^{\prime \prime}} \xi^{\prime}, \phi \xi\right) \\
& =-\bar{g}\left(\xi^{\prime \prime}, \bar{\nabla}_{\xi^{\prime}} \phi \xi\right)+\bar{g}\left(\xi^{\prime}, \bar{\nabla}_{\xi^{\prime \prime}} \phi \xi\right) \\
& =-\bar{g}\left(h^{l}\left(\xi^{\prime}, \phi \xi\right), \xi^{\prime \prime}\right)+\bar{g}\left(h^{l}\left(\xi^{\prime \prime}, \phi \xi\right), \xi^{\prime}\right) .
\end{aligned}
$$

On the other hand using $(2.4),(2.9),(2.11)$ and (2.14) we get

$$
\begin{aligned}
\bar{g}\left(\left[\xi^{\prime}, \xi^{\prime \prime}\right], \phi W\right) & =\bar{g}\left(\bar{\nabla}_{\xi^{\prime}} \xi^{\prime \prime}, \phi W\right)-\bar{g}\left(\bar{\nabla}_{\xi^{\prime \prime}} \xi^{\prime}, \phi W\right) \\
& =-\bar{g}\left(\phi \bar{\nabla}_{\xi^{\prime}} \xi^{\prime \prime}, W\right)+\bar{g}\left(\phi \bar{\nabla}_{\xi^{\prime \prime}} \xi^{\prime}, W\right) \\
& =-\bar{g}\left(\bar{\nabla}_{\xi^{\prime}} \phi \xi^{\prime \prime}, W\right)+\bar{g}\left(\bar{\nabla}_{\xi^{\prime \prime}} \phi \xi^{\prime}, W\right) \\
& =-\bar{g}\left(h^{s}\left(\xi^{\prime}, \phi \xi^{\prime \prime}\right), W\right)+\bar{g}\left(h^{s}\left(\xi^{\prime \prime}, \phi \xi^{\prime}\right), W\right),
\end{aligned}
$$

and

$$
\begin{aligned}
(4.11) \bar{g}\left(\left[\xi^{\prime}, \xi^{\prime \prime}\right], \phi N_{1}\right) & =-\bar{g}\left(\bar{\nabla}_{\xi^{\prime}} \phi \xi^{\prime \prime}-\left(\bar{\nabla}_{\xi^{\prime}} \phi\right) \xi^{\prime \prime}, N_{1}\right)+\bar{g}\left(\bar{\nabla}_{\xi^{\prime \prime}} \phi \xi^{\prime}-\left(\bar{\nabla}_{\xi^{\prime \prime}} \phi\right) \xi^{\prime}, N_{1}\right) \\
& =-\bar{g}\left(\nabla_{\xi^{\prime}} \phi \xi^{\prime \prime}, N_{1}\right)+\bar{g}\left(\nabla_{\xi^{\prime \prime}} \phi \xi^{\prime}, N_{1}\right) \\
& =-\bar{g}\left(h^{*}\left(\xi^{\prime}, \phi \xi^{\prime \prime}\right), N_{1}\right)+\bar{g}\left(h^{*}\left(\xi^{\prime \prime}, \phi \xi^{\prime}\right), N_{1}\right) .
\end{aligned}
$$

Thus from (4.7) - (4.11) the proof is complete.

Theorem 4.4. Let $M$ be a proper GCR-lightlike submanifold of an indefinite Sasakian manifold $\bar{M}$. Then $D_{1}$ is integrable if and only if

(i) $\nabla_{X}^{* t} \phi Y-\nabla_{Y}^{* t} \phi X \in \Gamma\left(D_{1}\right)$.

(ii) $\operatorname{Bh}(X, \phi Y)=B h(Y, \phi X)$

(iii) $A_{\phi Y}^{*} X=A_{\phi X}^{*} Y \quad$ and belongs to $\quad \Gamma\left[\left(\bar{D} \oplus \phi D_{2}\right) \perp D_{0}\right]$

for any $X, Y \in \Gamma\left(D_{1}\right)$.

Proof. For any $X, Y \in \Gamma \operatorname{Rad}(T M)$ using (2.14), we have

$$
\begin{gathered}
\bar{\nabla}_{X} \phi Y=\phi \bar{\nabla}_{X} Y \\
\bar{\nabla}_{X} Y=-\phi \bar{\nabla}_{X} \phi Y-\epsilon g\left(A_{Y}^{*} X, V\right) V \\
\nabla_{X} Y+h(X, Y)=-\phi\left(\nabla_{X} \phi Y+h(X, \phi Y)\right)-\epsilon g\left(A_{Y}^{*} X, V\right) V .
\end{gathered}
$$

Let $X, Y \in \Gamma\left(D_{1}\right)$ and using (2.10) we have

$$
\nabla_{X} Y+h(X, Y)=-\phi\left(-A_{\phi Y}^{*} X+\nabla_{X}^{* t} \phi Y+h(X, \phi Y)\right)-\epsilon g\left(A_{Y}^{*} X, V\right) V,
$$


equating the tangential components of above equation both sides, we get

$$
\left.\nabla_{X} Y=f A_{\phi Y}^{*} X-f \nabla_{X}^{* t} \phi Y-B h(X, \phi Y)\right)-\epsilon g\left(A_{Y}^{*} X, V\right) V
$$

replacing $X$ by $Y$ and subtracting resulting equation from this equation, we get

$$
\begin{aligned}
{[X, Y]=} & f\left(A_{\phi Y}^{*} X-A_{\phi X}^{*} Y\right)-f\left(\nabla_{X}^{* t} \phi Y-\nabla_{Y}^{* t} \phi X\right)+B h(X, \phi Y)-B h(Y, \phi X) \\
& -\epsilon g\left(A_{Y}^{*} X, V\right) V+\epsilon g\left(A_{X}^{*} Y, V\right) V,
\end{aligned}
$$

thus $[X, Y] \in \Gamma\left(D_{1}\right)$ if and only if $\nabla_{X}^{* t} \phi Y-\nabla_{Y}^{* t} \phi X \in \Gamma\left(D_{1}\right), B h(X, \phi Y)=$ $B h(Y, \phi X), A_{\phi Y}^{*} X=A_{\phi X}^{*} Y$ and belong to $\Gamma\left[\left(\bar{D} \oplus \phi D_{2}\right) \perp D_{0}\right]$, this completes the proof.

Theorem 4.5. Let $M$ be a proper GCR-lightlike submanifold of an indefinite Sasakian manifold $\bar{M}$. Then $D_{2}$ is integrable if and only if

(i) $\nabla_{X}^{*} \phi Y-\nabla_{Y}^{*} \phi X \in \Gamma\left(\phi D_{2}\right)$.

(ii) $\operatorname{Bh}(X, \phi Y)=B h(Y, \phi X)$.

(iii) $h^{*}(X, \phi Y)-h^{*}(Y, \phi X)$

(iv) $A_{X}^{*} Y$ and belongs to $\Gamma\left[\left(\bar{D} \oplus \phi D_{2}\right) \perp D_{0}\right]$, for any $X, Y \in \Gamma\left(D_{2}\right)$.

Proof. Let $X, Y \in \Gamma\left(D_{2}\right)$ and using (2.10) in (4.12)we have

$$
\nabla_{X} Y+h(X, Y)=-\phi\left(\nabla_{X}^{*} \phi Y+h^{*}(X, \phi Y)+h(X, \phi Y)\right)-\epsilon g\left(A_{Y}^{*} X, V\right) V
$$

equating the tangential components of above equation, we get

$$
\left.\nabla_{X} Y=-f \nabla_{X}^{*} \phi Y-f h^{*}(X, \phi Y)-B h(X, \phi Y)\right)-\epsilon g\left(A_{Y}^{*} X, V\right) V,
$$

replacing $X$ by $Y$ and subtracting resulting equation from this equation, we get

$$
\begin{aligned}
{[X, Y]=} & -f\left(\nabla_{X}^{*} \phi Y-\nabla_{Y}^{*} \phi X\right)-f\left(h^{*}(X, \phi Y)-h^{*}(Y, \phi X)\right)-B h(X, \phi Y) \\
& +B h(Y, \phi X)-\epsilon g\left(A_{Y}^{*} X, V\right) V+\epsilon g\left(A_{X}^{*} Y, V\right) V,
\end{aligned}
$$

thus $[X, Y] \in \Gamma\left(D_{2}\right)$ if and only if $\nabla_{X}^{*} \phi Y-\nabla_{Y}^{*} \phi X \in \Gamma\left(\phi D_{2}\right), h^{*}(X, \phi Y)=$ $h^{*}(Y, \phi X), B h(X, \phi Y)=B h(Y, \phi X), A_{X}^{*} Y$ and belongs to $\Gamma\left[\left(\bar{D} \oplus \phi D_{2}\right) \perp D_{0}\right]$, which proves the theorem.

Theorem 4.6. Let $M$ be a proper GCR-lightlike submanifold of an indefinite Sasakian manifold $\bar{M}$. Then $\phi D_{2}$ is integrable if and only if

(i) $g\left(A_{Y}^{*} \phi X, \phi Z\right)=g\left(A_{X}^{*} \phi Y, \phi Z\right)$

(ii) $h^{s}(\phi X, Y)=h^{s}(\phi Y, X)$

(iii) $\bar{g}\left(h^{l}(\phi X, Y), \xi\right)=\bar{g}\left(h^{l}(\phi Y, X)\right.$ 
(iv) $g\left(\phi Y, A_{N} \phi X\right)=g\left(\phi X, A_{N} \phi Y\right)$

for any $X, Y \in \Gamma\left(D_{2}\right), Z \in \Gamma\left(D_{0}\right), W \in \Gamma(S), N \in \Gamma(\operatorname{ltr}(T M))$, and $\xi \in \Gamma\left(D_{2}\right)$.

Proof. Using the definition of $G C R$-lightlike submanifolds, it is clear that $\phi D_{2}$ is integrable if and only if

$$
\begin{aligned}
\bar{g}([\phi X, \phi Y], Z) & =\bar{g}([\phi X, \phi Y], V)=\bar{g}([\phi X, \phi Y], \phi W) \\
& =\bar{g}([\phi X, \phi Y], \phi \xi)=\bar{g}([\phi X, \phi Y], N)=0,
\end{aligned}
$$

for any $X, Y \in \Gamma\left(D_{2}\right), Z \in \Gamma\left(D_{0}\right), W \in \Gamma(S), \xi \in \Gamma\left(D_{2}\right)$ and $N \in \Gamma(l \operatorname{tr}(T M))$.

From (2.2), (2.10)-(2.12), (2.13) and (2.14), we obtain

$$
\begin{aligned}
\bar{g}([\phi X, \phi Y], Z) & =\bar{g}\left(\bar{\nabla}_{\phi X} \phi Y, Z\right)-\bar{g}\left(\bar{\nabla}_{\phi Y} \phi X, Z\right) \\
& =\bar{g}\left(\phi \bar{\nabla}_{\phi X} Y, Z\right)-\bar{g}\left(\phi \bar{\nabla}_{\phi Y} X, Z\right) \\
& =-\bar{g}\left(\bar{\nabla}_{\phi X} Y, \phi Z\right)+\bar{g}\left(\bar{\nabla}_{\phi Y} X, \phi Z\right) \\
& =-g\left(\nabla_{\phi X} Y, \phi Z\right)+g\left(\nabla_{\phi Y} X, \phi Z\right) \\
& =g\left(A_{Y}^{*} \phi X, \phi Z\right)-g\left(A_{X}^{*} \phi Y, \phi Z\right),
\end{aligned}
$$

and

$$
\begin{aligned}
\bar{g}([\phi X, \phi Y], V) & =\bar{g}\left(\bar{\nabla}_{\phi X} \phi Y, V\right)-\bar{g}\left(\bar{\nabla}_{\phi Y} \phi X, V\right) \\
& =-\bar{g}\left(\phi Y, \bar{\nabla}_{\phi X} V\right)+\bar{g}\left(\phi X, \bar{\nabla}_{\phi Y} V\right) \\
& =-g\left(\phi Y, \phi^{2} X\right)+g\left(\phi X, \phi^{2} Y\right) \\
& =0 .
\end{aligned}
$$

Using (2.11), we obtain

$$
\begin{aligned}
\bar{g}([\phi X, \phi Y], \phi W) & =\bar{g}\left(\bar{\nabla}_{\phi X} \phi Y, \phi W\right)-\bar{g}\left(\bar{\nabla}_{\phi Y} \phi X, \phi W\right) \\
& =\bar{g}\left(\phi \bar{\nabla}_{\phi X} Y, \phi W\right)-\bar{g}\left(\phi \bar{\nabla}_{\phi Y} X, \phi W\right) \\
& =\bar{g}\left(\bar{\nabla}_{\phi X} Y, W\right)+\bar{g}\left(\bar{\nabla}_{\phi Y} X, W\right) \\
& =\bar{g}\left(h^{s}(\phi X, Y), W\right)-\bar{g}\left(h^{s}(\phi Y, X), W\right),
\end{aligned}
$$

and

$$
\begin{aligned}
\bar{g}([\phi X, \phi Y], \phi \xi) & =\bar{g}\left(\bar{\nabla}_{\phi X} \phi Y, \phi \xi\right)-\bar{g}\left(\bar{\nabla}_{\phi Y} \phi X, \phi \xi\right) \\
& =\bar{g}\left(\phi \bar{\nabla}_{\phi X} Y, \phi \xi\right)-\bar{g}\left(\phi \bar{\nabla}_{\phi Y} X, \phi \xi\right) \\
& =\bar{g}\left(\bar{\nabla}_{\phi X} Y, \xi\right)+\bar{g}\left(\bar{\nabla}_{\phi Y} X, \xi\right) \\
& =\bar{g}\left(h^{l}(\phi X, Y), \xi\right)-\bar{g}\left(h^{l}(\phi Y, X), \xi\right),
\end{aligned}
$$

finally

$$
\begin{aligned}
\bar{g}([\phi X, \phi Y], N) & =\bar{g}\left(\bar{\nabla}_{\phi X} \phi Y, N\right)-\bar{g}\left(\bar{\nabla}_{\phi Y} \phi X, N\right) \\
& =-\bar{g}\left(\phi Y, \bar{\nabla}_{\phi X} N\right)+\bar{g}\left(\phi X, \bar{\nabla}_{\phi Y} N\right) \\
& =\bar{g}\left(\phi Y, A_{N} \phi X\right)-\bar{g}\left(\phi X, A_{N} \phi Y\right) .
\end{aligned}
$$


Thus from (4.12)-(4.21) the proof is complete.

Theorem 4.7. Let $M$ be a GCR-lightlike submanifold of an indefinite Sasakian manifold $\bar{M}$. Then each leaf of radical distribution is totally geodesic in $M$ if and only if

(i) $A_{\xi}^{*} \xi^{\prime} \notin \Gamma\left(D_{0} \perp M_{1}\right)$

(ii) $\bar{g}\left(h^{s}\left(\xi^{\prime}, \phi \xi^{\prime \prime}\right), W\right)=0$

(iii) $\bar{g}\left(h^{*}\left(\xi^{\prime}, \phi \xi^{\prime \prime}\right), N_{1}\right)=0$,

where $M_{1}=\phi(L)$ for any $\xi \in \Gamma\left(D_{2}\right), \xi^{\prime}, \xi^{\prime \prime} \in \Gamma(\operatorname{Rad}(T M)), N_{1} \in \Gamma(L), W \in \Gamma(S)$ and $Z \in \Gamma\left(D_{0}\right)$.

Proof. Using the definition of $G C R$-lightlike submanifold of an indefinite Sasakian manifold, each leaf of $\operatorname{Rad}(T M)$ defines totally geodesic foliation in $M$ if and only if

$\bar{g}\left(\nabla_{\xi^{\prime}} \xi^{\prime \prime}, V\right)=\bar{g}\left(\nabla_{\xi^{\prime}} \xi^{\prime \prime}, Z\right)=\bar{g}\left(\nabla_{\xi^{\prime}} \xi^{\prime \prime}, \phi \xi\right)=\bar{g}\left(\nabla_{\xi^{\prime}} \xi^{\prime \prime}, \phi W\right)=\bar{g}\left(\nabla_{\xi^{\prime}} \xi^{\prime \prime}, \phi N_{1}\right)=0$, for any $\xi \in \Gamma\left(D_{2}\right), \xi^{\prime}, \xi^{\prime \prime} \in \Gamma(\operatorname{Rad}(T M)), N_{1} \in \Gamma(L), W \in \Gamma(S)$, and $Z \in \Gamma\left(D_{0}\right)$. From (2.4), (2.10) we obtain

$$
\begin{aligned}
g\left(\nabla_{\xi^{\prime}} \xi^{\prime \prime}, V\right) & =\bar{g}\left(\bar{\nabla}_{\xi^{\prime}} \xi^{\prime \prime}, V\right) \\
& =-\bar{g}\left(\xi^{\prime \prime}, \bar{\nabla}_{\xi^{\prime}} V\right) \\
& =-g\left(\xi^{\prime \prime},-\phi \xi^{\prime}\right) \\
& =0,
\end{aligned}
$$

and

$$
g\left(\nabla_{\xi^{\prime}} \xi^{\prime \prime}, Z\right)=-g\left(A_{\xi^{\prime \prime}}^{*} \xi^{\prime}, Z\right)
$$

and

$$
g\left(\nabla_{\xi^{\prime}} \xi^{\prime \prime}, \phi \xi\right)=-g\left(A_{\xi^{\prime \prime}}^{*} \xi^{\prime}, \phi \xi\right) .
$$

On the other hand using (2.4), (2.9), (2.11) and (2.14) we get

$$
\begin{aligned}
g\left(\nabla_{\xi^{\prime}} \xi^{\prime \prime}, \phi W\right) & =\bar{g}\left(\bar{\nabla}_{\xi^{\prime}} \xi^{\prime \prime}, \phi W\right) \\
& =-\bar{g}\left(\phi \bar{\nabla}_{\xi^{\prime}} \xi^{\prime \prime}, W\right) \\
& =-\bar{g}\left(\bar{\nabla}_{\xi^{\prime}} \phi \xi^{\prime \prime}, W\right) \\
& =-\bar{g}\left(h^{s}\left(\xi^{\prime}, \phi \xi^{\prime \prime}\right), W\right),
\end{aligned}
$$

and

$$
\begin{aligned}
g\left(\nabla_{\xi^{\prime}} \xi^{\prime \prime}, \phi N_{1}\right) & =\bar{g}\left(\bar{\nabla}_{\xi^{\prime}} \xi^{\prime \prime}, \phi N_{1}\right) \\
& =-\bar{g}\left(\phi \bar{\nabla}_{\xi^{\prime}} \xi^{\prime \prime}, N_{1}\right) \\
& =-\bar{g}\left(\bar{\nabla}_{\xi^{\prime}} \phi \xi^{\prime \prime}-\left(\bar{\nabla}_{\xi^{\prime}} \phi\right) \xi^{\prime \prime}, N_{1}\right) \\
& =-\bar{g}\left(\nabla_{\xi^{\prime}} \phi \xi^{\prime \prime}, N_{1}\right) \\
& =-\bar{g}\left(h^{*}\left(\xi^{\prime}, \phi \xi^{\prime \prime}\right), N_{1}\right) .
\end{aligned}
$$


Hence form (4.22) - (4.26), the assertion follows.

Theorem 4.8. Let $M$ be a GCR-lightlike submanifold of an indefinite Sasakian manifold $\bar{M}$. If $M$ is D-geodesic then each leaf of holomorphic distribution is totally geodesic foliation in $M$.

Proof. For $X, Y \in \Gamma(D)$ we have

$$
\begin{aligned}
h(X, Y) & =\bar{\nabla}_{X} Y-\nabla_{X} Y \\
& =\bar{\nabla}_{X}\left(-\phi^{2} Y+\eta(Y) V\right)-\nabla_{X} Y \\
& =-\left(\bar{\nabla}_{X} \phi\right)(\phi Y)-\phi \bar{\nabla}_{X} \phi Y-\nabla_{X} Y \\
& =\bar{g}(X, \phi Y) V-\phi \bar{\nabla}_{X} \phi Y-\nabla_{X} Y \\
& =\bar{g}(X, \phi Y) V-\phi \nabla_{X} \phi Y-\phi h(X, \phi Y)-\nabla_{X} Y \\
& =\bar{g}(X, \phi Y) V-f \nabla_{X} \phi Y-\omega \nabla_{X} \phi Y-B h(X, \phi Y)-C h(X, \phi Y)-\nabla_{X} Y .
\end{aligned}
$$

Equating the transversal parts both sides we get

$$
h(X, Y)=-\omega \nabla_{X} \phi Y-C h(X, \phi Y),
$$

$M$ is $D$-geodesic implies $-\omega \nabla_{X} \phi Y=0$. Thus the proof is complete.

Acknowledgements The authors would like to thank the anonymous referee for his/her comments that helped us to improve this article.

\section{References}

[1] V. I. Arnol'd, Contact geometry: the geometrical method of Gibbs's thermodynamodynamics, in Proceedings of the Gibbs Symposium (New Haven, CT, 1989), 163-179, american Mathematical Society, Providence, RI, USA, 1990.

[2] A. Bejancu, CR Submanifolds of a Kaehler Manifold I, Proc. Amer. Math. Soc., 69(1978), 135-142.

[3] A. Bejancu, CR Submanifolds of a Kaehler Manifold II, Trans. Amer. Math. Soc., 250(1979), 333-345.

[4] A. Bejancu, M. Kon and K. Yano, CR Submanifolds of a Complex Space Form, J. Diff. Geom., 16(1981), 137-145.

[5] B. Y. Chen, CR Submanifolds of a Kaehler Manifold I , J. Diff. Geom., 16(1981), 305-322.

[6] B. Y. Chen, CR Submanifolds of a Kaehler Manifold II, J. Differential Geom., 16(1981), 493-509.

[7] C. Calin, On Existence of Degenerate Hypersurfaces in Sasakian Manifolds, Arab Journal of Mathematical Sciences, 5(1999), 21-27. 
[8] K. L. Duggal, CR Structures and Lorentizian Geometry, Acta Appl. Math., 7(1986), 211-223.

[9] K. L. Duggal, Lorentzian Geometry of CR Submanifolds, Acta Appl. Math., 17(1989), 171-193.

[10] K. L. Duggal and A. Bejancu, Lightlike Submanifolds of semi-Riemannian Manifolds and Applications, Vol. 364 of Mathematics and its Applications, Kluwer Academic Publishers, Dordrecht, The Netherlands, (1996).

[11] K. L. Duggal, and B. Sahin, Lightlike Submanifolds of Indefinite Sasakian Manifolds, International Journal of Mathematics and Mathematical Sciences, Vol (2007), Article ID 57585, 21 pages.

[12] K. L. Duggal and B. Sahin, Generalized Cauchy-Riemann Lightlike Submanifolds of Indefinite Sasakian Manifolds, Acta Math. Hungar, 122(2009), 45-58.

[13] Rakesh Kumar, Rachna Rani and R. K. Nagaich, On Sectional curvature of $\epsilon$-Sasakian Manifolds, International Journal of Mathematics and Mathematical Sciences, Vol (2007), Article ID 93562, 8 pages.

[14] V. E. Nazaikinskii, V. E. Shatalov and B. Y. Sternin, Contact geometry of linear differential equations, Vol. 6 of De Gruyter Expositions in Mathematics, Walter de Gruyter, Berlin, Germany, 1992.

[15] S. Maclane, Geometrical Mechanics II, Lectures notes, University of Chicago, Chicago III, USA, 1968.

[16] K. Yano, and M. Kon, Differential Geometry of CR Submanifolds, Geometriae Dedicata, 10(1981), 369-391.

[17] K. Yano, and M. Kon, Contact CR-submanifolds, Kodai Math. J., 5(1982), 238-252.

[18] K. Yano, and M. Kon, CR Submanifolds of Kaehlerian and Sasakian Manifolds, Birkhauser, Bostan, (1983). 